\title{
REVIEW
}

\section{THE EMERGING ROLE OF IMMUNOTHERAPY IN GASTROESOPHAGEAL CANCER: STATE OF ART AND FUTURE PERSPECTIVE}

\author{
A. Raimondi ${ }^{1}$, M. Prisciandaro ${ }^{1,2}$, F. Pagani ${ }^{1}$, G. Randon ${ }^{1}$, F. Corti ${ }^{1}$, F. Nichetti ${ }^{1}$, \\ M. Niger ${ }^{1}$, F. Morano ${ }^{1}$, F. Pietrantonio', M. Di Bartolomeo' \\ ${ }^{1}$ Department of Medical Oncology, Fondazione IRCCS Istituto Nazionale dei Tumori, Milan, Italy \\ ${ }^{2}$ Department of Oncology and Hematology, University of Milan, Milan, Italy
}

\section{CORRESPONDING AUTHOR:}

Maria Di Bartolomeo

Department of Medical Oncology

Fondazione IRCCS Istituto Nazionale dei Tumori di Milano

via Venezian 1

20133 Milan, Italy

E-mail: maria.dibartolomeo@istitutotumori.mi.it

ORCID: 0000-0002-7954-6609

Doi: $10.48286 / a r o .2022 .34$

History

Received: Nov 14, 2021

Accepted: Jan 31, 2022

Published: Mar 1, 2022

\begin{abstract}
The introduction of immunotherapy in the therapeutic algorithm of gastroesophageal cancer is still a debated issue. Recent findings from randomized clinical trials documented the efficacy of adjuvant nivolumab in improving disease free survival (DFS) in resectable esophageal and gastroesophageal junction cancer patients with residual pathologic disease after neoadjuvant chemoradiation (CheckMate 577). Consistently, the combination of pembrolizumab and doublet chemotherapy with 5-fluorouracil plus cisplatin improved first-line treatment outcomes in metastatic esophageal squamous cancer; moreover the major benefit was observed in tumor expressing PD-L1 combined positive score (CPS) $>10$ (Keynote 590). Finally, the addition of nivolumab to first-line oxaliplatin and 5-fluorouracil-based chemotherapy improved overall surviv-
\end{abstract}

al, progression free survival and response rate in patients with metastatic gastric/gastroesophageal junction cancer with PD-L1 positive score (PD-L1 CPS $\geq 5$ ) (CheckMate 649). Moving forward, the research focused on the identification of predictive biomarkers of response to immunotherapy, to refine the patients' selection and maximize the treatment benefit. Microsatellite instability has been shown to predict higher response to checkpoint inhibitors as highlighted by subgroup analyses of the pivotal studies. For what concerns microsatellite stable tumors, the expression of PD-L1, the positivity for Epstein-Barr virus and a high tumor mutational burden are now regarded as the most promising and reliable predictive markers for immunotherapy as far as now. Therefore, the anti-PD1 agents nivolumab and pembrolizumab proved to confer 
an improvement in the outcome of gastroesophageal cancer patients but the real magnitude of benefit of immunotherapy in this disease setting is under definition. Biomarker-focused research will allow clinicians to define the optimal therapeutic algorithm in the different patients populations.

\section{KEY WORDS}

Immunotherapy; biomarker; gastric cancer; PD-L1; microsatellite instability.

\section{IMPACT STATEMENT}

Immune-checkpoint inhibitors proved to confer a meaningful benefit in the setting of gastric/gastroesophageal junction cancer, nevertheless a refinement of patients selection according to predictive biomarkers could maximize the treatment benefit.

acteristics of tumor, mainly the presence of HER2 overexpression/amplification, and on the other side, the clinical conditions and comorbidities of patients (11). The doublet combination with platinum derivative and fluoropyrimidine is considered a standard of care with or without trastuzumab (in case of HER2 overexpressed/amplified tumors). In the second-line setting and in later treatment lines, the combination of taxanes plus ramucirumab, ramucirumab monotherapy or irinotecan represent the main choices, even though with poor survival outcomes (12-17). The research progresses led to a deeper understanding of the molecular characterization of GC, providing the opportunity to classify tumors into different subtypes on the basis of their genomic profile, with the most common TCGA classification, furtherly described in the table I (18).

The immunotherapy revolution deeply changed the therapeutic management and the prognosis of patients in several cancer settings, such as non-small cell lung cancer (NSCLC), melanoma, renal cell carcinoma (RCC), urothelial cancer and head and neck tumors (19). The principal therapeutic weapon is represented by immune checkpoint inhibitors (ICls) targeting the programmed cell death receptor 1 and its ligand (PD1 and PD-L1). In fact, tumors upregulate the inhibitory checkpoints of the immune system, while ICls release the brakes and reactivate T-cells activity in order to promote the anti-tumor immune reaction(20). In the setting of GC/GEJC, several studies have been conducted or are ongoing to explore and define the potential role of ICls. In this review we aim at depicting a comprehensive picture of the current scenario and of the future perspectives. 


\section{LOCALIZED DISEASE}

In the setting of localized or locally advanced disease, eligible for curative radical surgery, few data have been collected on the role of immunotherapy. First of all, in stage II/III esophageal or GEJC patients treated with chemoradiotherapy followed by surgery and with evidence of residual disease, adjuvant treatment with nivolumab for 1 year provided a statistically significant and clinically meaningful advantage in disease-free survival (DFS) (median DFS 22.4 vs 11.0 months, HR 0.69, 95\% Cl 0.56-0.86, $\mathrm{p} \leq 0.001$ ) over placebo in the phase III CheckMate 577 trial. Disease-free survival favored nivolumab across multiple prespecified subgroups, and the benefit was more pronounced in the squamous histotype (median DFS 29.7 vs 11.0 months, HR $0.61,95 \% \mathrm{Cl} 0.42-0.88$ ) although maintained also in the adenocarcinoma subtype (median DFS 19.4 vs 11.1 months, HR $0.75,95 \% \mathrm{Cl} 0.59-0.96)$, potentially opening a new therapeutic scenario (21).

Moving forward, clinical trials are ongoing in order to provide evidence-based results. In details, the randomized, open-label, phase II DANTE study (NCT03421288) is investigating the combination of the anti-PD-L1 agent atezolizumab to peri-operative FLOT regimen (5fluorouracil, oxaliplatin and docetaxel), followed by adjuvant atezolizumab, versus standard peri-operative FLOT in GC or GEJC (Siewert I-III) CT2 or higher, any $\mathrm{N}$ or node positive, without any biological selection and HER2 status not assessed. The randomization is stratified per microsatellite instability (MSI) status while PD-L1 expression is performed but does not represent a stratification factor. The study completed the recruitment and the presented safety results showed that the chemo-immuno regimen was safe and feasible in the peri-operative setting of GC/ GEJC, while activity and efficacy results are not available yet (22). Similarly, the randomized, double-blind, phase III KEYNOTE-585 study (NCT03221426) is investigating pembrolizumab or placebo combined with peri-operative chemotherapy, followed by pembrolizumab or placebo maintenance in $\mathrm{T} 3$ or higher or $\mathrm{N}$ positive GC/GEJC patients. The initial chemotherapy schedule was cisplatin plus 5fluorouracil or capecitabine, but the study was amended to include a cohort with FLOT after the results of the FLOT4 trial (9). The trial will assess the status of MSI and PD-L1 as exploratory biomarkers, though neither MSI status nor PDL1 represent stratification factors. The two above-described trials are investigating immunotherapy in an unselected population. However, the results of the recent pivotal trials conducted in the metastatic setting highlighted how predictive biomarkers of response to immunotherapy are crucial to select patients with predicted enhanced response to ICls. Particularly, as discussed above, agnostic tumors with MSI-high status are highly responsive to immunotherapy, thus clinical trials are ongoing in this peculiarly selected subpopulation $(23,24)$. The rationale relies in the results of proof of concept studies that showed how pre-operative immunotherapy could achieve a pathologic major or complete response in potentially resectable mismatch repair deficient (dMMR)/MSI-high tumors and eventually provide a chance of cure even regardless

\section{SUBTYPE}

Chromosomal Instability (CIN)

Genomically Stable (GS)

Epstein-Barr Virus (EBV)

Microsatellite Instability (MSI),
$20 \%$

$9 \%$

\section{FREQUENCY}

$50 \%$

$22 \%$

\section{CHARACTERISTICS}

- Intestinal histology

- TP53 mutation

- High frequency of tyrosine kinase/RAS pathway activation

- Diffuse histology

- CDH1, RHOA mutations

- CLDN18-ARHGAP fusion

- alterations in cellular adhesion molecules genes

- PIK3CA mutation

- PD-L1/2 overexpression

- EBV-CIMP

- CDKN2A silencing

- Immune cell signalling

- Hypermutation

- Gastric-CIMP

- MLH1 silencing

- Mitotic pathways

Table I. Description of The Cancer Genome Atlas Classification (TCGA) of gastric cancer. 
of surgery. In details, in the phase II NICHE study, a window of opportunity treatment with 1 cycle of ipilimumab plus nivolumab in resectable colorectal cancer patients obtained no meaningful response in pMMR cases while a major or complete pathological response in all but one dMMR ones (25). This was confirmed by a case series of localized MSI-high GC or colon cancer patients achieving a high rate of $\mathrm{pCR}$ after immunotherapy (26). On this basis, two trials are ongoing to test immunotherapy in MSI-high GC/GEJC patients eligible for radical surgery. The first one is the GERCOR NEONIPIGA trial (NCT04006262) that is aimed at enrolling 32 patients to receive a 12-week preoperative combo-immunotherapy with nivolumab plus ipilimumab and, after radical surgery, postoperative nivolumab up to 1 year. The second one is the Italian, multicenter, single-arm, multicohort, phase II INFINITY study (NCT04817826) aimed at investigating the safety and activity of the ICls combination durvalumab (1500 mg q4w for 3 cycles) plus tremelimumab (300 $\mathrm{mg}$ single dose) as preoperative or potentially definitive treatment in dMMR/MSI-high/Epstein-Barr (EBV) negative GC/GEJC patients. The Cohort 1 is enrolling up to 18 patients and its primary endpoint is the rate of pCR at surgery after neoadjuvant immunotherapy, while Cohort 2 will investigate a non-operative-management strategy in patients achieving complete clinical response at radiological, tissue and liquid biopsy level after immunotherapy (figure 1) (27).

\section{UNTREATED METASTATIC DISEASE}

In the setting of first-line treatment for advanced/ metastatic GC/GEJC, the first study was the non-ran- domized, multicohort, phase II KEYNOTE-059 study that investigated pembrolizumab in combination with standard cisplatin-fluoropyrimidine chemotherapy irrespectively of PD-L1 expression in Cohort 2 and pembrolizumab monotherapy in patients with PD-L1 combined positive score (CPS) $\geq$ 1 in Cohort 3. Overall, 25 and 31 patients were enrolled in Cohort 2 and 3, respectively: the ORR was $60.0 \%(95 \% \mathrm{Cl}, 38.7-78.9)$ and $25.8 \%$ (95\% Cl 11.944.6), median duration of response was 4.6 and 9.6 months, and median overall survival (OS) was 13.8 and 20.6 months, respectively, with a globally manageable tolerability profile (28).

On this basis, the randomized, phase III KEYNOTE-062 trial was designed and conducted in treatment naïve advanced GC/GEJC Asian and non Asian patients selected for PD-L1 expression CPS $\geq 1$. A total of 763 patients were randomized 1:1:1 to pembrolizumab monotherapy versus pembrolizumab plus standard cisplatin-fluoropyrimidine chemotherapy versus placebo plus chemotherapy. The complex statistical design of the study compared pembrolizumab to placebo plus chemotherapy, showing the non-inferiority (primary endpoint) (median OS 10.6 vs 11.1 months, HR 0.91, $99.2 \% \mathrm{Cl} 0.69-1.18$ ) but not the superiority of pembrolizumab as compared to chemotherapy. Nevertheless, it should be pointed out that at least half of fit-for-a-trial patients treated with pembrolizumab died earlier than with chemotherapy and that the accepted confidence interval for inferiority margin worse than chemotherapy was wide, besides the absence of an improvement in quality of life. Moreover, the addition of pembrolizumab to chemotherapy failed to condition an improvement

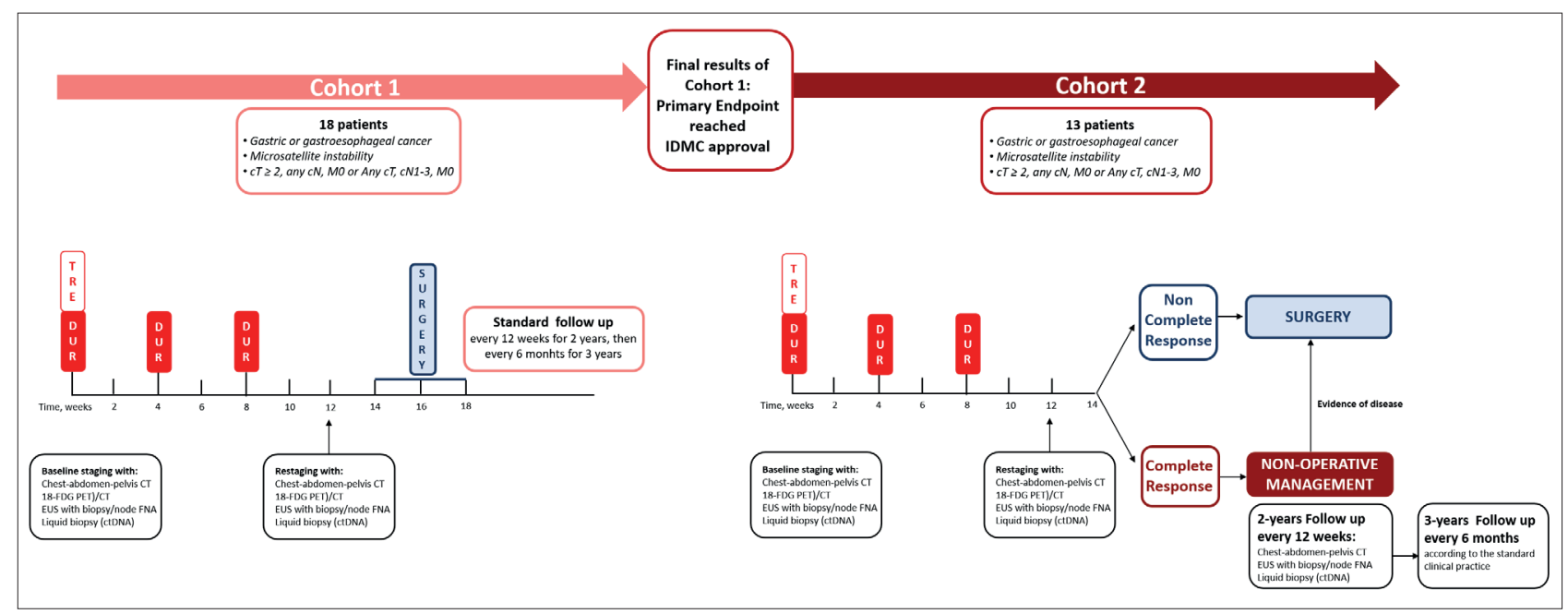

Figure 1. Study Diagram. 